\title{
Ferritina: biomarcador de doenças cardiovasculares em pacientes diabéticos
}

\section{Ferritin: a biomarker of cardiovascular diseases in diabetic patients}

\author{
Amanda Justi ${ }^{1}$, Pamela Tatsch², Luciano Oliveira Siqueira ${ }^{2}$
}

${ }^{1}$ Curso de Medicina, Faculdade de Medicina, Universidade de Passo Fundo (UPF) - Passo Fundo (RS), Brasil

${ }^{2}$ Curso de Farmácia, Instituto de Ciências Biológicas, Universidade de Passo Fundo (UPF) - Passo Fundo (RS), Brasil

DOI: https://dx.doi.org/10.7322/abcshs.v44i1.1145

\section{RESUMO}

Introdução: O Diabetes Mellitus (DM) é uma condição clínica caracterizada pela hiperglicemia por defeito na secreção e/ ou resistência à insulina. Objetivo: Verificar a correlação entre os marcadores de inflamação; Proteína C Reativa de alta sensibilidade (PCR-US), ferritina e lipídeos de pacientes diabéticos. Métodos: Foi realizada a análise do perfil lipídico de 50 pacientes diabéticos do tipo 2, com uma média de quatro anos de diagnóstico. Analisou-se a concentração plasmática de glicose, colesterol total, HDL colesterol, LDL colesterol e VLDL colesterol, triglicerídeos, ferritina e PCR-US. Resultados: Evidenciou uma correlação positiva forte entre PCR-US e ferritina $(r=0,85)$; entre ferritina e colesterol LDL e triglicerídeos $(r=0,91 ; r=0,91)$; correlação positiva moderada para colesterol total $(r=0,63)$; negativa moderada para colesterol HDL $(r=0,38)$. Conclusão: Os resultados indicam que a ferritina apresenta uma correlação positiva forte com a PCR-US, triglicerídeos e colesterol LDL, que, quando analisada de forma combinada, sugere a suspeita de processo inflamatório ativo e na ausência de doença de base, maior possibilidade de suspeita de inflamação aterosclerótica nestes pacientes.

Palavras-chave: Diabetes Mellitus; inflamação; proteína C-reativa; Ferritinas; aterosclerose.

\begin{abstract}
Introduction: Diabetes Mellitus (DM) is a clinical condition characterized by hyperglycemia default in secretion and/or insulin resistance. Objective: Check the correlation between markers of inflammation, high sensitivity C-reactive protein (hs-CRP), ferritin and lipids in diabetic patients. Methods: We carried out the analysis of lipid profiles of 50 type 2 diabetic patients, with an average of four years of diagnosis. We analyzed the plasma concentration of glucose, total cholesterol, HDL cholesterol, LDL cholesterol, VLDL cholesterol, triglycerides, ferritin and hs-CRP. Results: The study showed a strong positive correlation between ferritin and hs-CRP ( $r=0.85)$, between ferritin and LDL cholesterol and triglycerides $(r=0.91, r=0.91)$, moderate positive correlation to total cholesterol $(r=0.63)$; moderate negative for $\mathrm{HDL}$ cholesterol $(r=0.38)$. Conclusion: The results indicate that ferritin shows a strong positive correlation with hs-CRP, triglycerides and LDL cholesterol that, when considered in combination, suggest the suspicion of active inflammatory process and a greater chance of atherosclerosis in these patients.
\end{abstract}

Keywords: Diabetes Mellitus; inflammation; C-reactive protein; Ferritin; atherosclerosis.

Recebido em: 20/03/2018

Revisado em: 26/09/2018

Aprovado em: 25/10/2018

Autor para correspondência: Luciano de Oliveira Siqueira - Universidade de Passo Fundo, Instituto de Ciências Biológicas, Curso de Farmácia - BR 285 Km 171, Bairro São José - CEP: $99052-900$ - Passo Fundo (RS), Brasil - E-mail: Iuciano@upf.br

Conflito de interesses: nada a declarar. 


\section{INTRODUÇÃO}

O Diabetes Mellitus (DM) é um distúrbio metabólico caracterizado pela persistente elevação da glicose sérica - hiperglicemia, ocasionado deficiência da secreção de insulina pelas células-beta do pâncreas, resistência periférica à ação da insulina ou ambas. A hiperglicemia está associada a diversas complicações, tanto micro quanto macrovasculares, bem como ao aumento da morbi-mortalidade ${ }^{1,2}$.

A prevalência de DM e hipertensão está aumentando no mundo inteiro, seguindo maior frequência nos indivíduos com excesso de peso, o que está ligado a modificações na dieta, a qual se apresenta com uma oferta de alimentos de maior teor calórico, combinada a diminuição da atividade física. Nesse contexto, o Brasil tem implementado políticas importantes para a prevenção de diabetes, hipertensão e hiperlipidemias (doenças crônicas não transmissíveis), tendo sua mortalidade ajustada em 2010 por idade uma queda de $1,8 \%$ ao ano $^{3}$.

Nesse sentido, a resistência à insulina é considerada um importante fator de risco para doenças cardiovasculares, uma vez que a insulina tem sua ação parcialmente bloqueada, promove à elevação de triglicerídeos, redução do HDLcolesterol, promoção de um LDL-colesterol mais oxidado e denso, tornando-se mais aterogênico ${ }^{4}$.

Por conseguinte, a síndrome metabólica (SM), caracterizada por um conjunto de doenças com risco cardiovascular elevado, como hipertensão, obesidade, dislipidemias e hiperglicemias, cuja base é a resistência insulínica - está relacionada com o aumento da mortalidade. A SM atinge aproximadamente $85 \%$ dos pacientes com DM tipo 2 e se associa a uma prevalência crescente de complicações 5 .

Assim, defeitos da ação da insulina em tecidos-alvo (fígado, músculo e tecido adiposo) promovem a ascensão de um processo inflamatório crônico de baixa intensidade. Independentemente do agente desencadeador, o elo entre resistência insulínica (RI) e processo inflamatório é bidirecional, ou seja, qualquer inflamação crônica de baixa intensidade ocasionada pela obesidade induz RI e, essa, por sua vez, acentua o processo inflamatório num sistema retroalimentado ${ }^{1-6}$.

Nesse sentido, a inflamação envolve inúmeros tecidos e órgãos do corpo do indivíduo portador de DM. O tecido adiposo, principalmente abdominal, produz citocinas e substâncias bioativas, como TNF- $\alpha$, interleucinas, leptina, adiponectina, angiotensinogênio, entre outros; há aumento da infiltração de macrófagos e células do sistema imune nos tecidos; bem como pode haver uma inflamação das ilhotas pancreáticas. Esses processos inflamatórios podem tanto anteceder quanto estar associados à doença e, uma vez que essa inflamação sistêmica não é inerte, acarreta consequências ao organismo, tais como danos micro e macrovasculares ${ }^{7-9}$.

Nesse contexto, com a melhor compreensão da fisiopatologia da aterosclerose e dos eventos coronarianos agudos, evidenciou-se que a inflamação desempenha papel chave e que participa de todas as fases do processo aterosclerótico. Da mesma forma, os marcadores inflamatórios podem se tornar importantes ferramentas laboratoriais para estratificar e predizer eventos cardiovasculares. Dentre os marcadores inflamatórios estudados até o momento, a Proteína C Reativa de alta sensibilidade (PCR-US) é o que possui maior correlação com os eventos coronarianos e fornece informação prognóstica suplementar, independentemente dos fatores de risco tradicionais ${ }^{10}$.

A PCR-US, além de ser um biomarcador de lesão vascular relacionado à inflamação, primordialmente em placas de ateroma, é um relevante marcador de ativação endotelial. Assim, pode ser empregado como fator prognóstico em coronariopatias (angina e infarto do miocárdio), uma vez que acelera o processo de aterosclerose $\mathrm{e}^{11-13}$.

Em estudo com pessoas saudáveis, verificou-se uma correlação entre os níveis séricos de PCR-US e os componentes da síndrome metabólica, os quais são: glicemia de jejum, circunferência abdominal, triglicerídeos, HDL-colesterol, pressão arterial sistólica e diastólica, valores de insulina, índice de sensibilidade à insulina, colesterol total e LDL-colesterol ${ }^{12,13}$. Logo, os valores de PCR-US aumentam com as desordens metabólicas (dislipidemia, adiposidade central, RI e hipertensão). Assim, haja vista que a inflamação crônica subclínica inclui-se na síndrome metabólica, esta se torna um preditor bioquímico de eventos cardiovasculares - sendo o Reynolds Risk Score, por meio do uso sistemático da PCR-US, um importante adjuvante na análise de risco ${ }^{6,12}$.

No entanto, além da PCR-US, a ferritina - proteína envolvida no metabolismo do ferro, também pode ser um importante marcador inflamatório. O ferro é absorvido no intestino e transportado no soro em sua forma férrica ligado à transferrina, podendo entrar nos tecidos periféricos por meio de receptores específicos. Já o ferro na forma ferrosa é incorporado à ferritina, a qual é sintetizada nas células e também encontrada nos eritrócitos ${ }^{14}$.

Quando há infecções, traumatismos ou inflamações, a concentração da ferritina se eleva nas 24 a 48 horas iniciais, atingindo o pico no terceiro dia e mantendo-se aumentada por algumas semanas - é, portanto, um marcador de fase aguda ${ }^{13}$. Ainda não se sabe, contudo, como ocorre exatamente o mecanismo de aumento da ferritina na inflamação. Alguns estudiosos defendem que ela tem efeito anti-inflamatório, enquanto outros falam a favor do efeito pró-inflamatório ${ }^{15}$.

Dessa forma, a aterosclerose é reconhecida nos dias de hoje como doença inflamatória e a síntese de ferritina regulada por uma via alternativa, que é pelas citocinas pró-inflamatórias - como a interleucina-1, o aumento da ferritina pode ser considerado um marcador de inflamação e, consequentemente, de aterosclerose $\mathrm{e}^{16-18}$.

Em um estudo realizado por González et al. ${ }^{16}$, quando descartadas outras causas de inflamação, verificou-se a correlação positiva 
entre ferritina e resistência insulínica, baseado no modelo de avaliação de Homeostasis Model Assessment (HOMA). O HOMA, por meio de uma amostra de glicemia e de insulina obtidas em jejum, busca evidenciar a associação entre a capacidade pancreática de produzir insulina e manter os níveis glicêmicos a partir de um cálculo matemático ${ }^{17,18}$. Ryan et al. ${ }^{19}$, também demonstrou uma relação positiva entre ferritina, resistência insulínica e ácidos graxos em mulheres pré-menopausa, sugerindo que o metabolismo do ferro exerce grande influência na obesidade.

Entre os parâmetros de inflamação, estudos têm mostrado a relação entre a elevação dos valores da Proteína C Reativa de alta sensibilidade, um reagente de fase aguda, um marcador sensível de processo inflamatório subclínico e a resistência insulínica, igualmente com cada um dos componentes associados à síndrome metabólica. A elevação dos valores de PCR-US prevê o desenvolvimento de DM e doenças cardiovasculares. No entanto, estudos que associam valores de ferritina com parâmetro inflamatório de risco da doença cardiovascular em pacientes diabéticos ainda são escassos ${ }^{11}$.

Partindo destas premissas, o objetivo desse estudo foi avaliar a correlação entre PCR-US, ferritina e lipídeos de pacientes diabéticos como forma de desenvolvimento de marcadores bioquímicos para a prevenção, acompanhamento e cuidado nas doenças cardiovasculares.

\section{MÉTODOS}

\section{Delineamento}

O estudo é transversal sobre a correlação entre lipídeos, PCRUS e ferritina de pacientes diabéticos tipo 2. Em atendimento aos aspectos éticos legais de pesquisa envolvendo seres humanos, o projeto foi submetido ao Comitê de Ética em Pesquisa (CEP) da Universidade de Passo Fundo, sendo aprovado sob $n^{\circ}$ de registro: 0099.0.398.000-11.

\section{Casuística}

Participaram do estudo 50 indivíduos diabéticos do tipo 2 do sexo masculino, com média de idade de $61 \pm 8$ anos; índice de massa corporal de $29,1 \pm 3,2 \mathrm{~km} / \mathrm{m}^{2}$.

\section{Critérios de inclusão e exclusão}

Foram incluídos aleatoriamente 50 indivíduos diabéticos do tipo 2 do sexo masculino, no mínimo quatro anos de evolução da doença, em tratamento no grupo de apoio ao paciente diabético e cadastrados no ambulatório da Faculdade de Medicina da Universidade de Passo Fundo, sem sinais e sintomas de doença inflamatória de base. Optou-se pela inclusão somente indivíduos do sexo masculino em razão da diferença hormonal, sexo e de massa muscular poderia influenciar no perfil lipídico e na concentração parâmetros do metabolismo do ferro - como a ferritina - e, assim, buscou-se evitar um viés de confusão.

Foram excluídos do estudo, os pacientes com menos de 50 anos, do sexo feminino, que apresentavam sinais ou sintomas de doença inflamatória de base, com menos de quatro anos de evolução de diabetes e/ou que não aceitaram participar do estudo.

\section{Modelo experimental}

A primeira parte do estudo compreendeu o preenchimento de um formulário contendo a identificação dos pacientes. Posteriormente, em uma sala de coleta (reservada no ambulatório da Faculdade de Medicina), na primeira hora da manhã e após um período de $12 \mathrm{~h}$ de jejum, foram coletadas, assepticamente, amostras de $10 \mathrm{~mL}$ de sangue venoso mediante punção na fossa antecubital. A partir disso, uma alíquota de $2 \mathrm{~mL}$ de sangue foi anticoagulada com EDTA $2 \mathrm{mg} / \mathrm{dL}$ para determinação de hemoglobina glicada e o restante do sangue foi centrifugado a 3453 rpm por 10 minutos (2000 g - força centrífuga relativa). O soro foi extraído e acondicionado em frascos Eppendorff para posterior análise bioquímica num período máximo de uma hora após a coleta. A análise bioquímica constituiu da determinação de triglicerídeos, colesterol total, HDL-colesterol e glicose (Labtest); as determinações de VLDL, LDL-colesterol foram obtidas mediante cálculo pela equação de Friedwald; hemoglobina glicada total foi determinada pelo método de Cromatografia de troca iônica (Labtest). A determinação da proteína $\mathrm{C}$ reativa de alta sensibilidade e ferritina se deram pelo método de imunoturbidimetria (Labtest). Todos os testes foram realizados de acordo com as especificações dos fabricantes. Após a execução técnica, as concentrações dos analitos foram determinadas em analisador bioquímico semi-automático TP Analyzer Plus (Thermoplate, China).

\section{Análise estatística}

Os dados foram testados quanto a sua normalidade mediante análise de Shapiro-Wilk. A seguir, os resultados foram compilados para análise estatística descritiva. Para análise de correlação entre os parâmetros foi utilizado o teste de correlação de Spearman para dados não paramétricos ou de Pearson para os paramétricos, no pacote estatístico do SPSS 22.0 considerando $\mathrm{p}<0,05$ como nível mínimo de significância.

A partir dos dados compilados, procedeu-se análise de correlação em comparação de postos, que se consiste em ordenar os elementos que compõem os dois conjuntos de valores em análise, calculando-se então o coeficiente de correlação de Spearman. Resultados expressos em número absoluto onde:

- 0,9 positivo ou negativo indica uma correlação muito forte;

- 0,7 a 0,9 positivo ou negativo indica uma correlação forte;

- 0,5 a 0,7 positivo ou negativo indica uma correlação moderada;

- 0,3 a 0,5 positivo ou negativo indica uma correlação fraca;

- 0 a 0,3 positivo ou negativo indica uma correlação desprezível. 


\section{RESULTADOS}

Os pacientes diabéticos mostraram uma hiperglicemia leve, com glicose de 146,1 $\pm 9,8 \mathrm{mg} / \mathrm{dL}$ e hemoglobina glicada de $8,8 \pm 0,4 \%$. Relacionado ao perfil lipídico, os valores de triglicerídeos, colesterol total, LDL-colesterol, VLDL foram: 136,5 $\pm 15,3$ $\mathrm{mg} / \mathrm{dL} ; 188,8 \pm 6,1 \mathrm{mg} / \mathrm{dL} ; 121,1 \pm 4,7 \mathrm{mg} / \mathrm{dL}$; e $29,3 \pm 2,8 \mathrm{mg} /$ $\mathrm{dL}$, respectivamente, enquanto os valores de ferritina foram $210,2 \pm 6,7 \mu \mathrm{g} / \mathrm{L}$ e os de PCR-US foram 4,7 $\pm 0,6 \mathrm{mg} / \mathrm{L}$. (Tabela 1 ).

A análise de correlação de Spearman (Figura 1) para dados não paramétricos evidenciou uma forte correlação positiva entre PCRUS e ferritina $(0,85)$, moderada correlação positiva de PCR-US com colesterol total e LDL-colesterol $(0,39$ e 0,49$)$, respectivamente, e fraca correlação positiva com triglicerídeos séricos $(0,12)$. Isso indica que a elevação da ferritina tem uma ligação direta (forte) com a elevação da PCR-US; moderada com colesterol total e LDL-colesterol e fraca para triglicerídeos. Por outro lado, apresentou uma moderada correlação negativa com glicose $(-0,57)$, HDL-colesterol $(-0,40)$ e hemoglobina glicada $(-0,36)$ indicando que a elevação de ferritina exprime uma relação inversamente proporcional ao aumento de glicose, hemoglobina glicada e HDL-colesterol (Figura 1).
A análise dos resultados mostra uma correlação positiva forte e estatisticamente significante $(\mathrm{p}<0,01)$ entre ferritina com LDLcolesterol e triglicerídeos $(0,91 ; 0,91)$, respectivamente (Figura 2), além de uma correlação positiva e estatisticamente significante $(\mathrm{p}<0,05)$ moderada para colesterol total $(0,63)$; negativa moderada para HDL-colesterol $(-0,38)$ e positiva fraca comparada a glicose $(0,22)$. Os resultados mostram também uma correlação negativa forte e estatisticamente significante $(\mathrm{p}<0,01)$ entre ferritina e hemoglobina glicada $(\mathrm{p}<0,01)$ (Figura 2$)$.

\section{DISCUSSÃO}

Conforme Salonen et al. ${ }^{20}$, altos níveis de ferro foram encontrados em homens finlandeses de meia-idade, medidos por meio de ferritina. Nesse estudo, valores de ferritina superiores a $200 \mu \mathrm{g} / \mathrm{L}$, aumentam 2,2 vezes mais o risco de desenvolvimento de um infarto agudo do miocárdio. Além disso, diversos estudos epidemiológicos têm apoiado a teoria de que os níveis elevados de ferro armazenados aumentam o risco de doença arterial coronariana (DAC). Baseado nisso, os pacientes com valores de ferritina iguais

Tabela 1: Biomarcadores inflamatórios, perfil lipídico e glicemia da população estudada.

\begin{tabular}{|l|c|c|c|}
\hline Parâmetro bioquímico & Média \pm Desvio Padrão & Coeficiente de variação & Valores de referência \\
\hline PCR-US (mg/L) & $4,7 \pm 0,6$ & $3,9 \%$ & Até $3 \mathrm{mg} / \mathrm{L}$ \\
\hline Hemoglobina glicada $(\%)$ & $8,8 \pm 0,4$ & $4,5 \%$ & $7,0-8,0 \mathrm{Meta}$ terapêutica para D.M. \\
\hline VLDL $(\mathrm{mg} / \mathrm{dL})$ & $29,3 \pm 2,8$ & $13,4 \%$ & $>40 \mathrm{mg} / \mathrm{dL}$ \\
\hline HDL-colesterol $(\mathrm{mg} / \mathrm{mL})$ & $38,5 \pm 1,8$ & $4,0 \%$ & $<130 \mathrm{mg} / \mathrm{dL}$ \\
\hline LDL-colesterol $(\mathrm{mg} / \mathrm{mL})$ & $121,1 \pm 4,7$ & $7,8 \%$ & $<150 \mathrm{mg} / \mathrm{dL}$ \\
\hline Triglicerídeos $(\mathrm{mg} / \mathrm{dL})$ & $136,5 \pm 15,3$ & $13,4 \%$ & $70 \mathrm{a} 100 \mathrm{mg} / \mathrm{dL}$ \\
\hline Glicose $(\mathrm{mg} / \mathrm{dL})$ & $146,1 \pm 9,8$ & $0,9 \%$ & $<190 \mathrm{mg} / \mathrm{dL}$ \\
\hline Colesterol $(\mathrm{mg} / \mathrm{dL})$ & $188,8 \pm 6,1$ & $8,1 \%$ & 30 a $220 \mu \mathrm{mg} / \mathrm{L}$ \\
\hline Ferritina $(\mu \mathrm{g} / \mathrm{L})$ & $210,2 \pm 6,7$ & $0,03 \%$ & \\
\hline
\end{tabular}

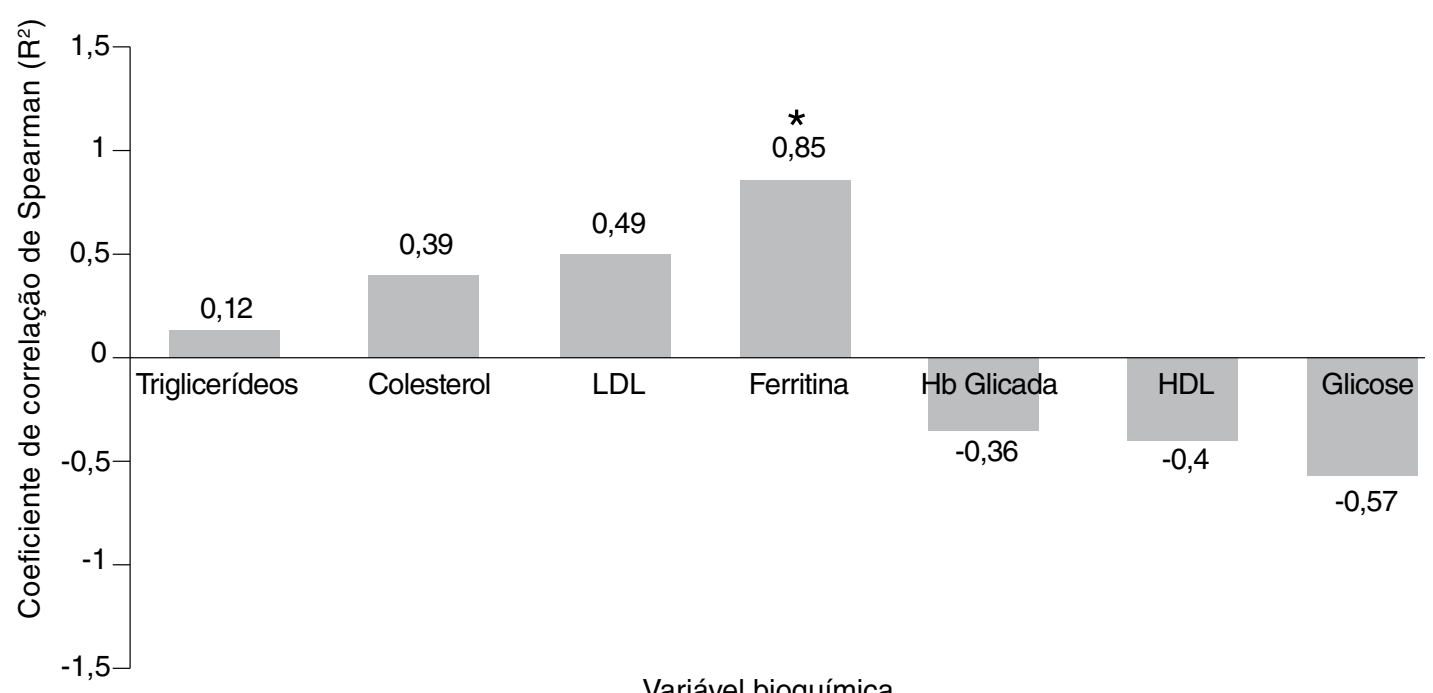

Variável bioquímica

Figura 1: Correlação linear entre a concentração de PCR-US com lipídeos, ferritina e glicemia na população estudada. Valores expressos com coeficiente de correlação de Spearman $\left(R^{2}\right) .\left({ }^{*} p<0,05\right)$ 
a $208,8 \pm 6,9 \mu \mathrm{g} / \mathrm{L}$ em nosso estudo já estariam sob um risco elevado de desenvolvimento de doenças cardiovasculares.

Kiechl et al. ${ }^{21}$ relataram que a concentração de ferritina era altamente correlacionada com o espessamento da parede das artérias carótidas em um estudo de coorte longitudinal. Já Haidari et al. ${ }^{22}$, demonstraram, recentemente, que a ferritina está significativamente associada com um aumento do risco de DAC em homens iranianos. Baseado nos dados aqui elencados, o aumento do risco de DAC se deve pelo fato de que a ferritina pode contribuir com a diminuição do HDL-colesterol e com o aumento do LDL-colesterol.

Em estudo realizado por González et al. ${ }^{16}$, descartaram-se inúmeras causas de inflamação, identificando uma significativa correlação positiva entre ferritina e resistência insulínica, determinado pelo modelo de avaliação de homeostase (HOMA) e avaliando vários critérios de síndrome metabólica, especialmente o peso corporal, glicemia e triglicerídeos séricos.

Segundo estudo realizado por You et al. ${ }^{18}$, a ferritina de cadeia leve é cerca de 1,9 vezes maior em artérias coronarianas comprometidas do que em condições normais, sendo essa diferença significativa quando comparada ao sexo ou idade e conforme o tecido da autopsia ou transplante do paciente a ser analisado.

Nessa perspectiva, a dosagem da concentração plasmática de PCR-US - mais sensível marcador para avaliação de estados inflamatórios - ajuda a avaliar clinicamente a presença, a extensão e a atividade de um processo inflamatório, assim como auxilia no monitoramento da evolução e da resposta terapêutica. Normalmente, a PCR-US está presente em baixos valores $(<5 \mathrm{mg} / \mathrm{L})$ no sangue de pessoas normais, todavia a sua concentração pode aumentar de cem a mil vezes na vigência de processos inflamatórios, quando começa a ser secretada cerca de 6 horas após o quadro de inflamação $0^{6,10,18}$.

Pode-se dizer, portanto, que os dados obtidos são corroborados pelos achados prévios da literatura em relação à ferritina e seu papel em processos inflamatórios. Por tratar-se de um ensaio piloto, não houve estratificação entre sexos e idade, contudo, uma vez que o foco deste estudo foi avaliar a correlação entre os marcadores. Assim sendo, a utilização da ferritina como adjuvante para o prognóstico de doenças cardiovasculares mostrou-se relativamente confiável quando associada à determinação de PCR-US, de forma a aumentar a sensibilidade de marcadores bioquímicos na prevenção e diagnóstico de doenças cardiovasculares.

Logo, considerando as doenças cardiovasculares um problema de grande relevância para a saúde pública, sua prevenção, acompanhamento e cuidado são essenciais, e esses marcadores inflamatórios podem contribuir nesse sentido.

Ao mesmo tempo em que os resultados mostraram-se promissores em uma população diabética aleatória, o estudo limita-se por não ser uma população tão homogênea. Por conseguinte, estudos futuros e ensaios randomizados com uma população mais uniforme (sexo, idade, e variáveis antropométricas) poderão consolidar a utilidade da ferritina como adjuvante do diagnóstico e acompanhamento de risco de eventos cardiovasculares. Há de se ponderar ainda que a ferritina apresenta uma vantagem: não sofre alterações tão intensas em processos infecciosos, como a PCR-US, a qual já é um marcador inflamatório amplamente utilizado.

Além disso, o estudo limita-se por determinar a concentração de hemoglobina glicada por cromatografia de troca iônica (método não é certificado pelo National Glycohemoglobin

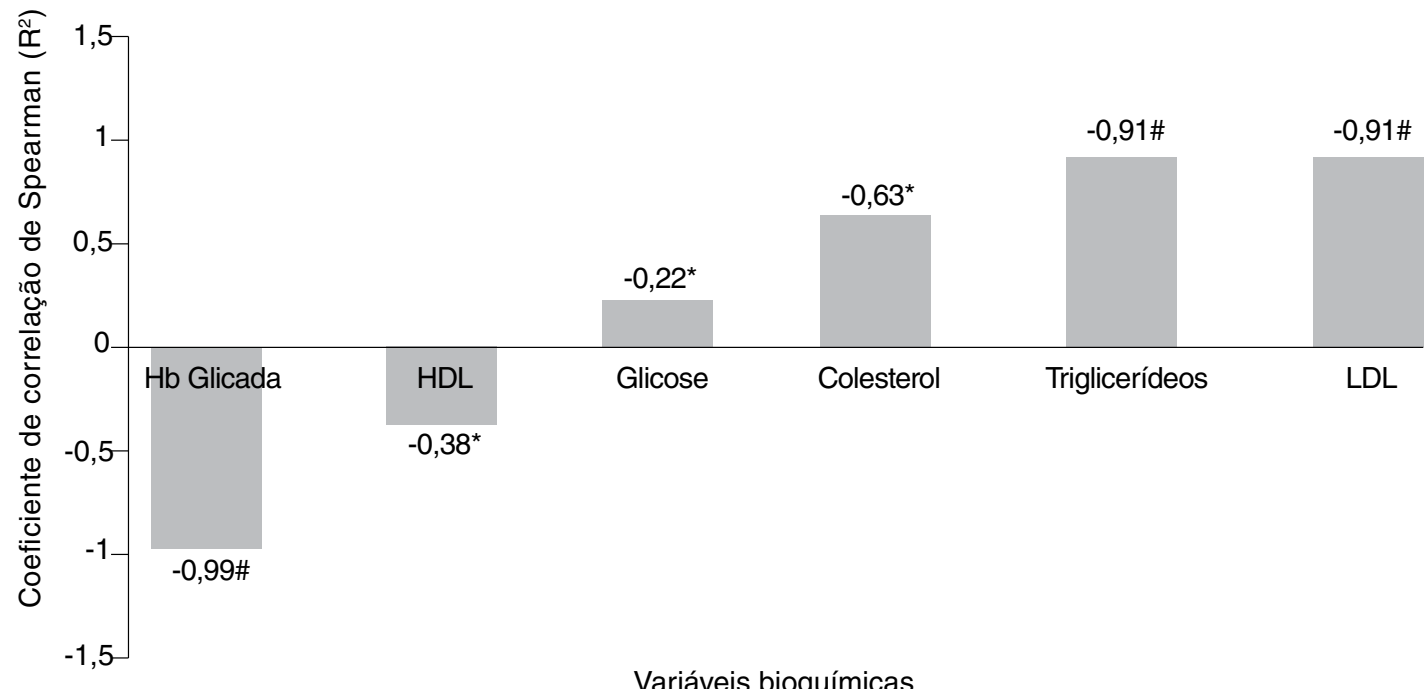

Figura 2: Correlação linear entre a concentração de ferritina com lipídeos e glicemia na população estudada. Valores expressos com coeficiente de correlação de Spearman $\left(R^{2}\right) .\left({ }^{*} p<0,05\right) ;\left({ }^{*} p<0,01\right)$ 
Standardization Program - NGSP), no entanto, por ser mais barata, a técnica é amplamente realizada em laboratórios de pequenas cidades. Na impossibilidade de realização de técnicas certificadas pelo NGSP e/ou por HPLC (restrita a maiores centros), para fins de acompanhamento destes pacientes nestas circunstancias é ainda uma ferramenta aceitável (desde que não se compare resultados de técnicas diferentes), uma vez que representa a glicemia do último trimestre.
Em conclusão, os dados obtidos nesse estudo apontam que a ferritina apresenta uma forte correlação positiva com PCR-US, triglicerídeos e LDL-colesterol. Quando analisada em conjunto com essas dosagens, pode servir como um importante adjuvante bioquímico para análise de risco e prevenção de doenças cardiovasculares, sendo, portanto, de grande relevância no cuidado e acompanhamento de doenças crônicas não transmissíveis - como o diabetes mellitus, de modo a advertir e evitar morbimortalidade.

\section{REFERÊNCIAS}

1. Fernandes MPA, Pace AE, Zanetti ML, Foss MC, Donadi EA. Fatores imunogenéticos associados ao diabetes mellitus do tipo 1. Rev Latino-Am Enfermagem. 2005;13(5):743-9. http://dx.doi.org/10.1590/S0104-11692005000500020

2. Sociedade Brasileira de Diabetes (SBD). Diretrizes da Sociedade Brasileira de Diabetes: 2017-2018. São Paulo: Clannad, 2017.

3. Schmidt IM, Duncan BB, Silva GA, Menezes AM, Monteiro CA, Barreto SM, et al. Chronic non-communicable diseases in Brazil: burden and current challenges. Lancet. 2011;377(9781):1949-61.

http://dx.doi.org/10.1016/S0140-6736(11)60135-9

4. Festa A, D'Agostino R Jr, Howard G, Mykkänen L, Tracy RP, Haffner SM. Chronic subclinical inflammation as part of the insulin resistance syndrome: the insulin resistance atherosclerosis study (IRAS). Circulation. 2000;102(1):42-47. https://dx.doi.org/10.1161/01.cir.102.1.42

5. Moehlecke M, Leitão CB, Kramer CK, Rodrigues TC, Nickel C, Silveiro SP, et al. Effect of metabolic syndrome and of its individual components on renal function of patients with type 2 diabetes mellitus. Braz J Med Biol Res. 2010;43(7):687-93.

6. Volp ACP, Alfenas RCG, Costa NMB, Minim VPR, Stringueta PC, Bressan J. Capacidade dos biomarcadores inflamatórios em predizer a síndrome metabólica. Arq Bras Endocrinol Metab. 2008;52(3):537-49

http://dx.doi.org/10.1590/S0004-27302008000300015

7. Lontchi-Yimagou E, Sobngwi E, Matsha TE, Kengne AP. Diabetes Mellitus and Inflammation. Curr Diab Rep. 2013;13(3)435-44. http://dx.doi.org/10.1007/s11892-013-0375-y

8. Calle MC, Fernandez ML. Inflammation and type 2 diabetes. Diabetes Metab. 2012;38(3):183-91.

http://dx.doi.org/10.1016/j.diabet.2011.11.006

9. van Greevenbroek MM, Schalkwijk CG, Stehouwer CD. Obesityassociated low-grade inflammation in type 2 diabetes mellitus: causes and consequences. Neth J Med. 2013;71(4);174-87.

10. Pithan E, Martins OMO. Marcadores inflamatórios e de disfunção endotelial: qual o papel na predição de risco. Rev Soc Cardiol RS. 2010; 18(20):1-11.

11. González AS, Guerrero DB, Soto MB, Díaz SP, del Olmo MM, Vidal O. Síndrome metabólico e inflamación. Clin Invest Arterioscl. 2006;18(3):89-95. http://dx.doi.org/10.1016/S0214-9168(06)73667-1

12. Cook NR, Paynter NP, Eaton CB, Manson JE, Martin LW, Robinson JG, et al. Comparison of the Framingham and Reynolds Risk scores for global cardiovascular risk prediction in the multiethnic Women's Health Initiative. Circulation. 2012;125(14):1748-56.

http://dx.doi.org/10.1161/CIRCULATIONAHA.111.075929

13. Rosa Neto NS, Carvalho JF. O uso de provas de atividade inflamatória em reumatologia. Rev Bras Reumatol. 2009;49(4):413-30. http://dx.doi.org/10.1590/S0482-50042009000400008

14. Kell DB, Pretorius E. Serum ferritin is as important inflammatory disease marker, as it is mainly a leakage product from damaged cells. Metallomics. 2014;6(4):748-73. http://dx.doi.org/10.1039/c3mt00347g

15. Gomes AC, Moreira AC, Mesquita G, Gomes MS. Modulation of Iron Metabolism in Response to Infection: Twists for All Tastes. Pharmaceuticals. 2018;11(3):E84. http://dx.doi.org/10.3390/ph11030084

16. González AS, Guerrero DB, Soto MB, Díaz SP, Martinez-Olmos $\mathrm{M}$, Vidal $\mathrm{O}$. Metabolic syndrome, insulin resistance and the inflammation markers C-reactive protein and ferritin. Eur $\mathrm{J}$ Clin Nutr. 2006:60(6):802-9.

http://dx.doi.org/10.1038/sj.ejcn.1602384

17. Almeida CA, Pinto AP, Ricco RG, Pepato MT, Brunetti IL. Determination of glycemia and insulinemia and the Homeostasis model assessment (HOMA) in schoolchildren and adolescents with normal body mass index. J Pediatr. 2008;34(2):136-40. http://dx.doi.org/10.2223/JPED.1767

18. You SA, Archacki SR, Angheloiu G, Moravec CS, Rao S, Kinter M, et al. Proteomic approach to coronary atherosclerosis shows ferritin light chain as a significant marker: evidence consistent with iron hypothesis in atherosclerosis. Physiol Genomics. 2003;13(1):25-30. http://dx.doi.org/10.1152/physiolgenomics.00124.2002

19. Ryan BJ, Van Pelt DW, Guth LM, Ludzki AC, Gioscia-Ryan RA, Ahn $\mathrm{C}$, et al. Plasma ferritin concentration is positively associated with in vivo fatty acid mobilization and insulin resistance in obese women. Exp Physiol. 2018. http://dx.doi.org/10.1113/EP087283

20. Salonen JT, Nyyssönen K, Korpela H, Tuomilehto J, Seppänen R, Salonen R. et al. High stored iron levels are associated with excess risk of myocardial infarction in eastern Finnish men. Circulation. 1992;86(3):803-11.

21. Kiechl S, Willeit J, Egger G, Poewe W, Oberhollenzer F, et al. Body iron stores and the risk of carotid atherosclerosis: prospective results from the Bruneck study. Circulation. 1997;96(10):3300-7. https://dx.doi.org/10.1161/01.CIR.96.10.3300

22. Haidari M, Javadi E, Sanati A, Hajilooi M, Ghanbili J. Association of increased ferritin with premature coronary stenosis in men. Clin Chem. 2001;47(9):1666-72. 